\title{
Politique
}

\section{Les politiques canadiennes de l'industrie aérospatiale}

\section{Robert Dalpé}

Numéro 8, automne 1985

Innovations et politiques technologiques

URI : https://id.erudit.org/iderudit/040499ar

DOI : https://doi.org/10.7202/040499ar

Aller au sommaire du numéro

Éditeur(s)

Société québécoise de science politique

ISSN

0711-608X (imprimé)

1918-6584 (numérique)

Découvrir la revue

Citer cet article

Dalpé, R. (1985). Les politiques canadiennes de l'industrie aérospatiale. Politique, (8), 103-129. https://doi.org/10.7202/040499ar d'utilisation que vous pouvez consulter en ligne.

https://apropos.erudit.org/fr/usagers/politique-dutilisation/ 


\title{
LES POLITIQUES CANADIENNES DE L'INDUSTRIE AÉROSPATIALE*
}

\author{
Robert Dalpé \\ Université de Montréal
}

Les politiques canadiennes de l'industrie aérospatiale font actuellement l'objet de contestation. Les sociétés d'État sont au cœur de la polémique. Chez Canadair et De Havilland, les prévisions de vente ne se sont pas concrétisées et elles ont été sérieusement atteintes par l'effrondrement du marché de l'aéronautique civile en 1982. Face aux problèmes de l'industrie au début des années soixante-dix, la solution du gouvernement canadien avait été de nationaliser ces entreprises. Dix ans plus tard, il est question de la privatisation de ces deux sociétés d'État. Les subventions versées à Bell Helicopter pour l'implantation de son usine québécoise et l'engagement non respecté de McDonnell Douglas en ce qui concerne les retombées industrielles du F-18 sont aussi d'autres facteurs de contestation.

Ces critiques renvoient d'abord à la question du rôle de l'État dans l'économie. Au-delà de ce débat, il importe de rechercher les causes structurelles des problèmes de l'industrie aérospatiale canadienne. En raison de l'organisation internationale de l'industrie et du mode d'insertion de l'industrie canadienne dans l'économie

\footnotetext{
* Je remercie Frances Anderson pour ses commentaires.
} 
internationale, le développement de l'industrie canadienne ne se fait pas sans contraintes. L'objectif de cet article est de développer cette proposition. Ainsi, il faut voir que la spécialisation dans la haute technologie, les créneaux et les marchés internationaux peut entraîner une grande instabilité de l'industrie. Nous recherchons principalement les contraintes qui découlent de ses stratégies technologiques et qui déterminent en partie quels marchés leur seront accessibles.

La première étape est de présenter l'organisation internationale de la production. Ensuite, on peut analyser la structure de l'industrie canadienne et les politiques gouvernementales qui s'y appliquent afin de saisir son mode d'insertion à l'économie internationale. On peut ainsi saisir les principaux problèmes auxquels cette industrie fait face, de même que les contraintes qui limitent ses possibilités de développement.

\section{Structure de l'industrie aérospatiale occidentale}

L'industrie aérospatiale comprend trois groupes de produits: les avions, les hélicoptères et les satellites. Les deux premiers groupes renvoient à l'industrie aéronautique ${ }^{1}$. Les entreprises actives dans le domaine des satellites ne forment pas une branche clairement identifiable. On y retrouve quelques firmes de l'aéronautique, mais aussi des firmes actives dans divers segments de l'industrie électronique. L'industrie des satellites connaît une croissance très rapide. Avec la militarisation de l'espace, ce groupe devient de plus en plus stratégique et son importance industrielle s'accrôtt.

1. Lorsqu'il est question de l'industrie aéronautique, nous désignons les firmes actives dans l'entretien ou la fabrication des avions et des hélicoptères. Les données présentées dans ce texte ne concernent quelquefois que l'industrie aéronautique parce que les organismes chargés de recueillir les statistiques (Statistique Canada et Census of U.S.) n'ont pas encore développé une catégorie intégrant les trois groupes. 
Afin de connaître la structure de l'industrie aérospatiale, on peut présenter ses principales caractéristiques.

Haute technologie. L'industrie aéronautique représente historiquement l'apparition de la haute technologie. Cette mutation s'est faite à la fin des années quarante suite aux développements technologiques découlant des impératifs de la guerre. Au début des années soixante-dix, quelques branches de l'industrie chimique et de l'industrie électrique ont aussi connu la même mutation. La haute technologie suppose que la concurrence porte de plus en plus sur la technologie: c'est-à-dire que l'innovation devient le premier facteur dans la compétition internationale. Les innovations se suivent à un rythme accéléré faisant en sorte que les produits et les techniques de production deviennent rapidement obsolètes. Également, dans une entreprise de haute technologie, une partie de l'activité de l'entreprise est directement orientée vers les activités de recherche, comptabilisées sous formes de dépenses et personnel en recherche et développement (R-D) ${ }^{2}$. Il ne faut pas extrapoler ces tendances à toutes les activités des entreprises et à toutes les entreprises de ces branches en ce sens que certaines productions restent peu intensives en technologie. $\grave{A}$ un autre niveau, la haute technologie est un phénomène politique, créant une demande d'aide gouvernementale. La compétition portant sur la technologie, les entreprises tentent de rendre leurs activités de recherche industrielle plus visibles afin de s'attirer le soutien des gouvernements. Autour de l'industrie militaire, s'est formé un groupe d'entreprises et de techniciens qui survit grâce à cette course à l'innovation.

Présence de l'État. L'industrie aérospatiale à travers le monde fonctionne en relation directe avec l'État. Cette relation est très visible en ce qui concerne les marchés militaires: l'État est l'unique

2. Le temps écoulé entre, d'une part, le lancement du projet, l'innovation et la fabrication du prototype et, d'autre part, la fabrication en série de l'appareil et son utilisation commerciale est de plus en plus long. Le risque est alors accru (Morvan, 1983:257). 
client de ces firmes et, de plus, il définit les produits et participe directement à l'innovation (Ponssard et Pouvourville, 1982). Les décisions de l'État en matière d'armement ont un impact immédiat sur les firmes et sur la structure de l'industrie. L'État joue aussi un rôle dans la production civile du fait que celle-ci s'appuie directement sur la production militaire (Mowery et Rosenberg, 1982). Les innovations se font d'abord dans la production militaire pour répondre aux besoins de l'État. Suite à ces développements technologiques, découlant de la participation financière et technique de l'État, l'innovation est ensuite utilisée dans la production civile.

Oligopole américain. Si la capacité concurrentielle de l'industrie américaine décline dans certaines branches, l'industrie aérospatiale américaine conserve toujours le leadership et domine confortablement l'industrie des pays industrialisés (Banque Paribas, 1982). Un indicateur de cette domination est la balance commerciale américaine de matériel aérospatial. En 1984, les exportations américaines de matériel aérospatial ont représentées 15,1 milliards $\$$, alors que les importations totalisaient 4,7 milliards $\$$, soit moins que le tiers des exportations (U.S, D.O.C., 1985). Il est également à noter que les importations ne représentent que $6 \%$ de la production américaine.

L'industrie américaine est aussi fortement concentrée. Les sept plus grandes firmes de l'industrie aéronautique occidentale sont américaines: United Technologies, Boeing, Rockwell, General Electric, McDonnell Douglas, Lockheed et General Dynamics. On retrouve ensuite deux firmes européennes: la firme anglaise British Aerospace et la firme française Aérospatiale. La fabrication des moteurs est faite au sein d'un oligopole fermé comprenant United Technologies et General Electric.

La domination américaine se perpétue grâce au soutien de l'État. Les politiques militaires de l'actuel gouvernement ont permis la relance de l'industrie aérospatiale américaine. Les carnets 
de commandes des firmes américaines ne sont comparables à ceux d'aucune entreprise concurrente dans un autre pays. Si McDonnell Douglas doit livrer plus de $1600 \mathrm{~F}-18$ à travers le monde (dont plus de 1350 à l'armée américaine), le Mirage-2000, de production française, ne peut espérer être fabriqué à plus de 350 exemplaires (Usine Nouvelle, 1984). Les firmes américaines ont alors traversé la crise du marché de l'aéronautique civile de 1982 grâce aux commandes militaires. Toutes les grandes firmes prennent part à des projets de renouvellement de la flotte américaine. Par exemple, Sikorsky ${ }^{3}$ doit livrer plus de 1000 hélicoptères Black Hawk, Pratt \& Whitney ${ }^{4}$ fabrique les moteurs des F-15 et F-16 pendant que General Electric fabrique les moteurs des Bombardiers B-1.

C'est ainsi grâce à la taille de leur marché national et au soutien technologique des laboratoires gouvernementaux que les firmes américaines conservent leur leadership. Ces firmes sont celles qui créent les nouvelles générations d'avion et qui mettent l'innovation sur le marché. Des firmes d'autres pays parviennent à développer des modèles, mais doivent recourir, pour les pièces demandant une plus grande expertise, aux pièces américaines. C'est ainsi que l'Airbus européen est équipé d'un moteur américain de General Electric.

Émergence de nouveaux concurrents. Cette présence des goùvernements est aussi visible dans les autres pays, mais elle suit d'autres stratégies. Ces interventions prennent souvent la forme de contrôle du commerce des produits aéronautiques. C'est-à-dire que plusieurs pays exigent un contenu national lorsqu'ils achètent des modèles américains. Quelques pays tentent aussi de se doter d'une industrie aérospatiale nationale en débutant la fabrication des produits de bas de gamme. Dès que les gouvernements ont

3. Sikorsky, le plus grand fabricant mondial d'hélicoptères, est une filiale de United Technologies.

4. Pratt \& Whitney est aussi une filiale de United Technologies. 
des marchés nationaux d'une certaine taille, ils décident d'appliquer l'une de ces stratégies. Les firmes de l'oligopoles vont alors participer à la fabrication des appareils dans ces nouveaux pays concurrents 5 . Il faut voir que ces exigences s'appliquent à tous les fabricants, faisant en sorte que les grandes multinationales américaines sont mieux placées pour répondre à ces demandes que les firmes nationales des autres pays.

La concurrence s'est accrue pour les produits de bas de gamme depuis que plusieurs pays soutiennent une industrie nationale. En plus du Royaume-Uni, de la France, de l'Allemagne et du Canada, de nouveaux fabricants sont entrés récemment sur le marché. Il s'agit notamment de l'Espagne, de l'Indonésie et du Brésil. La production de ces pays s'appuie encore largement sur la technologie américaine. Les multinationales acceptent de participer au développement de ces entreprises nationales dans la mesure où l'opération est rentable et leur leadership n'est aucunement en jeu. Les firmes de l'oligopole ne vendent pas la technologie en tant que telle, mais vendent plutôt des pièces incorporant cette technologie. Le développement de ces industries nationales représente alors un nouveau marché. En ce qui concerne le marché de ces nouvelles entreprises nationales, elles produisent généralement des appareils non fabriqués par les multinationales ou les vendent sur des marchés qui ne sont pas accessibles aux firmes de l'oligopole en raison des barrières commerciales.

Coopération internationale. La production de matériel aérospatial passe de plus en plus par la coopération entre les pays. À cet égard, les exemples les plus typiques sont le Concorde et l'Airbus ${ }^{6}$.

5. La firme allemande MBB, avant de s'impliquer dans un projet de fabrication d'hélicoptères avec une entreprise canadienne, avait participé à des projets similaires au Japon, aux Philippines et en Indonésie.

6. Airbus est issu de la coopération de la France (Airbus SNIAS, à 37,9\%) de l'Allemagne (MBB et UFW, à 37,9\%) du Royaume-Uni (British Aerospace, à 20,0\%) et l'Espagne (Casa, à 4,2 \%). 
Face au risque et à l'ampleur des investissements en recherche industrielle, la coopération permet de répartir les coûts entre plusieurs pays (Mytelka, 1983). On assiste alors à des regroupements autour d'une "firme leader» (Morvan, 1983: 264). Comme on le verra par la suite, trois des appareils concurrents du Dash-8 de De Havilland sont issus de la coopération entre deux pays.

Dans l'industrie aérospatiale, l'existence de ces accords de coopération entre les États a pour objectif de créer un marché plus vaste afin de combler l'écart par rapport au marché national des firmes américaines. La domination des Etats-Unis se fait toujours sentir parce que ces firmes fournissent toujours les pièces technologiquement plus complexes. Plusieurs pays peuvent faire démarrer leur industrie aérospatiale s'ils peuvent s'assurer la participation des multinationales américaines. Ces industries nationales peuvent utiliser les entreprises américaines dans la mesure où leur gouvernement peut financer les investissements et assurer les risques.

\section{L'industrie canadienne}

La présentation de l'industrie canadienne se fait d'abord en situant les principaux fabricants et le mode d'intégration à l'économie internationale. Ensuite, on présente deux indicateurs de sa capacité concurrentielle. Premièrement, la capacité technologique de l'industrie est déterminante de sa compétitivité dans la mesure où il s'agit d'une industrie de la haute technologie. Deuxièmement, puisqu'il s'agit d'une industrie où l'État est très présent, le soutien gouvernemental contribue à la compétitivité des firmes. Autrement dit, sans le soutien de l'État ou des investissements en recherche industrielle, il n'est pas possible d'avoir une industrie concurrentielle sur les marchés internationaux. 


\section{La structure de l'industrie ${ }^{7}$}

En 1984, les livraisons de l'industrie aérospatiale canadienne se chiffraient à 3,28 milliards\$ et on y employait 41000 personnes $^{8}$. A titre de comparaison, les livraisons de l'industrie américaine étaient de 80,1 milliards\$ et on y occupait 715000 personnes (U.S., D.O.C., 1985). L'industrie canadienne se classe au cinquième rang, derrière les États-Unis, la France, le Royaume-Uni et l'Allemagne'. Sa production est surtout destinée à l'exportation: en 1984, 80\% des livraisons ont été vendues hors du Canada.

L'industrie canadienne connaît un haut niveau de contrôle étranger. En 1980, 55\% des livraisons de l'industrie aéronautique provenaient d'entreprises qui étaient la propriété d'intérêts américains ou étrangers. Il est à noter que le contrôle canadien sur la branche a augmenté à partir du début des années soixante-dix jusqu'en 1978 avec la nationalisation de De Havilland et de Canadair, puis la relance de cette dernière depuis 1976. Il décroît cependant depuis 1978 et devrait continuer de le faire à cause de la réduction des activités chez les deux grandes firmes étatisées, la croissance de Pratt \& Whitney et l'arrivée de nouveaux concurrents étrangers, dont Bell Helicopter.

L'industrie canadienne s'appuie d'abord sur trois grandes entreprises. Pratt \& Whitney, filiale de la multinationale américaine United Technologies, est le plus important fabricant mondial de petits moteurs à turbine. La filiale détient des mandats mondiaux pour le développement et la fabrication de ces moteurs, qui sont exportés à $90 \%$ surtout vers les États-Unis. Pratt \& Whitney

7. Pour connaitre l'industrie aérospatiale canadienne, on peut consulter: Canada, Conseil des Sciences (1982), Canada, M.E.E.R. (1981) et Canada, Rapport du Comité ... (1983). Les études suivantes portent sur l'industrie aérospatiale québécoise: Québec, M.I.C.T.(1982), Québec, M.T.(1983). et Douglas Whitehead Consultants (1983).

8. D'après le rapport annuel de l'Association des industries aérospatiales du Canada. Statistiques présentées dans Canada. A. et S. (mai 1985).

9. La production américaine représente environ $70 \%$ de la production mondiale, alors que la production canadienne ne représente que $3 \%$. 
effectue un peu plus du quart des livraisons de l'industrie aérospatiale canadienne. Cette filiale est actuellement la firme la plus importante de l'industrie tant par sa taille que par ses activités de recherche.

On retrouve ensuite les deux firmes étatisées. D'un côté, de Havilland se spécialise dans la fabrication d'aéronefs commerciaux ou, plus spécifiquement, d'appareils court-courriers. L'entreprise a acquis une expertise dans le domaine des appareils à décollage et atterissage courts. De Havilland met sur le marché trois modèles court-courriers: le Twin-Otter, le Dash-8 et le Dash-7. La plupart des appareils sont équipés de moteurs provenant de Pratt \& Whitney. L'entreprise fabrique aussi un appareil militaire (le Buffalo DHC5). On y emploie environ 4000 personnes.

De son côté, Canadair a pour principale activité la fabrication du Challenger CL-600 et CL-601, tous deux équipés de moteurs américains. Canadair poursuit aussi la fabrication de l'avion-citerne CL-215 et d'engins de reconnaissance téléguidés. Finalement, Canadair fait de la sous-traitance, participant à la fabrication des Boeing 767 et 747-SP, des C-5A et Orion P-3C de Lockheed, des F-15 et F-18 de McDonnell Douglas, des EF-11 de Grumman et F-5 de Northrop. Cette entreprise emploie environ 5000 personnes. Ces deux firmes nationalisées réduisent leurs activités depuis quelques années.

Une firme canadienne, Spar Aerospace, connaît une croissance rapide et emploie maintenant plus de 2500 personnes. Celle-ci se situe dans la production en pleine expansion de matériel aérospatial et des satellites de télécommunications canadiens ANIK. Le développement rapide de cette entreprise peut être attribué à la politique canadienne des télécommunications en vigueur depuis les années cinquante. Spar Aerospace profite directement de la participation du gouvernement canadien au programme spatial américain ${ }^{10}$.

10. Spar Aerospace a fabriqué le bras (CANADARM) de la navette spatiale. 
Quelques entreprises de plus petite taille fabriquent aussi des produits spécialisés. Elles occupent généralement des créneaux sur le marché nord-américain. Elles sont donc le premier fabricant nord-américain de leur produit, sinon le seul. Ceci ne signifie nullement qu'il s'agisse automatiquement de grandes entreprises. On retrouve entre autres la firme sous contrôle canadien CAE Electronics qui est le premier fabricant mondial de simulateurs de vol pour les appareils civils et qui emploie 2200 travailleurs (Canada, M.E.E.R., 1981: 78). Une firme sous contrôle américain, Litton Systems, est spécialisée dans les systèmes de navigation à inertie et en est d'ailleurs le premier fabricant mondial. Une dizaine de firmes au Canada se retrouvent dans la même position.

La grande majorité des autres entreprises font de la soustraitance. Quelques grandes entreprises en font en totalité ou en partie: McDonnel Douglas, Boeing du Canada, Bristol Aerospace et Canadair. Sauf exceptions, les petites entreprises sont limitées à la sous-traitance. D'après certaines estimations, cette production occupe près de 5000 personnes (Canada, Rapport du Comité ..., 1983: 4. 11). Ces entreprises travaillent pour les fabricants canadiens, mais surtout pour les fabricants américains: elles sont généralement rattachées aux programmes des multinationales américaines. Le plus grand contrat de sous-traitance est détenu par McDonnel Douglas et concerne la fabrication du DC-9 de la société mère américaine. Dans certains cas, il s'agit s'assurer au Canada les retombées industrielles négociées au moment des achats du gouvernement canadien. Une activité connexe à la sous-traitance est la réparation et l'entretien des appareils. Cette activité emploie aussi quelques milliers de travailleurs.

La plupart des firmes étrangères actives dans ce secteur ont des mandats mondiaux. C'est le cas notamment de Pratt $\&$ Whitney, Bell Helicopter, McDonnell Douglas, Canadian Marconi, Garrett et Litton Systems. Il s'agit, d'une part, des entreprises étrangères fabriquant des produits spécialisés ou, d'autre part, de firmes 
faisant de la sous-traitance mais qui ont l'exclusivité de la fabrication pour certaines pièces.

\section{L'industrie canadienne face à l'industrie occidentale}

L'industrie aérospatiale canadienne est fortement intégrée à l'industrie américaine. Trois indicateurs permettent de situer le degré d'intégration. Premièrement, près de la moitié de la production canadienne est faite par des firmes sous contrôle américain. Cependant, il faut signaler que certaines filiales sont parmi les entreprises les plus innovatrices de la branche. Ces filiales ne sont généralement pas des répliques miniatures de leur maison-mère: certaines sont spécialisées, font de la recherche industrielle et ont une marge d'autonomie par rapport à la maison-mère. Cette situation provient surtout de la politique des mandats mondiaux du gouvernement canadien. Les activités de ces filiales sont intégrées à l'intérieur du réseau intra-firme nord-américain.

Deuxièmement, la production canadienne est fortement orientée vers le marché américain. De 1980 à 1983, près des trois quarts des exportations canadiennes étaient destinées aux ÉtatsUnis (tableau 1). Pendant la même période, au moins la moitié de la production canadienne était vendue sur le marché américain. C'est donc dire que la production se fait d'abord en fonction des Etats-Unis où l'industrie trouve son premier marché. Ceci signifie aussi que les aéronefs fabriqués au Canada sont développés en fonction des possibilités de vente sur le marché américain. Egalement, les pièces d'aéronefs sont vendues dans une large mesure aux firmes américaines faisant en sorte que l'industrie canadienne devient un fournisseur des multinationales américaines

Troisièmement, la technologie utilisée au Canada provient en grande partie des États-Unis. En ce qui concerne l'industrie aéronautique québécoise, on peut voir que ses sources de technologie sont, sauf exceptions, américaines (Boismenu, Dalpé et Ducaten- 
TABLEAU 1

Production et Exportation de matériel aéronautique $(000 \$)$

\begin{tabular}{|c|c|c|c|c|c|}
\hline & \multirow{2}{*}{ Livraisons } & \multirow{2}{*}{ Exportations } & \multirow{2}{*}{$\begin{array}{c}\text { Exportations } \\
\text { aux USA } \\
\%\end{array}$} & \multirow{2}{*}{$\frac{\text { Exportations }}{\begin{array}{c}\text { Livraisons } \\
\%\end{array}}$} & \multirow{2}{*}{ 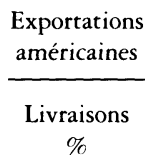 } \\
\hline & & & & & \\
\hline 1965 & 448312 & 207037 & 79 & 46 & 36 \\
\hline 1970 & 578198 & 379102 & 63 & 66 & 41 \\
\hline 1975 & 775125 & 421605 & 73 & 54 & 40 \\
\hline 1976 & 786178 & 453480 & 61 & 58 & 35 \\
\hline 1977 & 812666 & 485647 & 68 & 60 & 41 \\
\hline 1978 & 1145656 & 691001 & 68 & 60 & 41 \\
\hline 1979 & 1570990 & 1005499 & 71 & 64 & 45 \\
\hline 1980 & 2016360 & 1403402 & 74 & 70 & 52 \\
\hline 1981 & 2720238 & 1796527 & 76 & 66 & 50 \\
\hline 1982 & 2429385 & 1732125 & 69 & 71 & 49 \\
\hline 1983 & 1698077 & 1521006 & 78 & 90 & 70 \\
\hline
\end{tabular}

Source: Statistique Canada, Stocks, livraisons et commandes des industries manufacturières. Cat. 31-001: Industries manufacturières du Canada: niveaux national et provincial. Cat. 31-203: Sommaire du commerce extérieur, Cat. 65-(001; Exportations par marchandises, Cat. 65-004.

zeiler, 1985). La fabrication du Challenger de Canadair s'est aussi faite à partir d'une technologie américaine: les moteurs sont américains, puis les ailes et les réacteurs sont aussi de conception américaine.

Cette intégration semble devenir de plus en plus forte. C'est ainsi que la part que représente les exportations américaines dans la production canadienne s'est accrue continuellement depuis vingt ans (tableau 1). En effet, les exportations américaines représentaient le tiers de la production il y a vingt ans, alors qu'elles en représentent maintenant la moitié. La production canadienne est de plus en plus destinée aux marchés étrangers et surtout au marché américain.

Cette orientation de l'industrie canadienne peut s'expliquer par la taille du marché américain. Le Canada y est d'ailleurs le 
premier importateur. En 1983, 34\% des importations provenaient du Canada, comparativement à 29\% en 1978 (tableau 2). Face à la taille du marché américain, les importations canadiennes sont mineures, représentant environ $2 \%$ de la production américaine. $\mathrm{Si}$, au Canada, ces exportations sont stratégiques, aux États-Unis, elles sont presque négligeables.

\section{TABLEAU 2}

Importations américaines en matériel aéronautique $(000 \$)$

\begin{tabular}{lrr}
\hline \multirow{2}{*}{ Importateur } & \multicolumn{2}{c}{ Année } \\
\cline { 2 - 3 } & \multicolumn{1}{c}{1978} & 1983 \\
\hline Canada & 182969 & 691793 \\
Royaume-Uni & 141599 & 365686 \\
France & 126416 & 344076 \\
Japon & 30526 & 138132 \\
Italie & 29010 & 102216 \\
RFA & 25714 & 99173 \\
Israël & 28563 & 59108 \\
Pays-Bas & 580 & 45605 \\
Belgique-Luxembourg & 1834 & 40036 \\
Brésil & 1338 & 31074 \\
Singapour & 41041 & 28513 \\
Norvège & 1651 & 22983 \\
Espagne & 3397 & 22746 \\
Danemark & 3641 & 22401 \\
Australie & 13359 & 13103 \\
\multicolumn{1}{c}{ Autres } & 5556 & 24693 \\
\hline \multicolumn{1}{c}{ Total } & 637194 & 2051338 \\
\hline \multicolumn{1}{c}{} & & \\
\hline
\end{tabular}

Source: U.S., Bureau of Census, U.S. General Imports. Washington, U.S. Department of Commerce, 1978 et 1983.

Cette orientation s'explique aussi par la capacité industrielle canadienne dans cette branche. C'est-à-dire que l'industrie ne peut assurer entièrement le développement et la fabrication d'un appareil de grande taille. En ce sens, il faut s'appuyer sur la technologie 
américaine. À cet égard, la politique canadienne est alors de s'allier à l'industrie américaine et de profiter de son avance technologique. Cette orientation peut apparaître valable dans la mesure où l'industrie américaine peut conserver son leadership sur l'industrie occidentale.

En ce qui concerne les autres marchés, l'industrie canadienne se plaint de l'arrivée de nouveaux concurrents (Canada, Rapport

TABLEAU 3

Concurrents du Dash-8 de De Havilland

\begin{tabular}{lll}
\hline Avions & Pays & Fabricant du moteur \\
\hline Dash-8 & Canada & Pratt \& Whitney (PW' 120) \\
SD-360 & Royaume-Uni & Pratt \& Whitney (PT6A) \\
SF-340 & États-Unis et & General Electric \\
& Suède & \\
ATR-42 & France et Italie & Pratt \& Whitney (PW 120) \\
EMB-120 & Brésil & Pratt \& Whitney (PW 120) \\
CN-235 & Espagne et & General Electric \\
& Indonésie & \\
\hline
\end{tabular}

TABLEAU 4

Exportations canadiennes de moteurs et pièces de moteur en aéronautique - 1982-84 $(000 \$)$

\begin{tabular}{|c|c|c|c|c|c|c|}
\hline \multirow{3}{*}{ Pays } & \multicolumn{6}{|c|}{ Année } \\
\hline & \multicolumn{2}{|c|}{1982} & \multicolumn{2}{|c|}{1983} & \multicolumn{2}{|c|}{1984} \\
\hline & Rang & Montant & Rang & Montant & Rang & Montant \\
\hline États-Unis & 1 & 360329 & 1 & 336242 & 1 & 471651 \\
\hline Royaume-Uni & 4 & 20970 & 2 & 30772 & 2 & 34200 \\
\hline Brésil & 2 & 30708 & 3 & 24368 & 3 & 30308 \\
\hline Italie & 3 & 25515 & 4 & 22045 & 4 & 24128 \\
\hline Allemagne & 6 & 14289 & 5 & 15574 & 5 & 19269 \\
\hline Tous les pays & & 502916 & & 486834 & & 646386 \\
\hline
\end{tabular}

Source: Statistique Canada. Exportations par marchandises, Cat. 65-004. 
du Comité..., 1982: 1.2). Ceci reste valable pour les fabricants d'aéronefs. Cependant il faut signaler qu'une fraction de l'industrie canadienne approvisionne ces nouveaux concurrents. Par exemple, Pratt \& Whitney fournit des moteurs à trois concurrents du Dash8 de De Havilland (tableaux 3 et 4). En ce sens, une fraction de l'industrie canadienne profite aussi de l'arrivée de ces nouveaux concurrents. Ce sont souvent les entreprises qui ont la plus faible capacité technologique qui sont les plus touchées. Cet exemple illustre aussi le pouvoir de l'oligopole face aux fabricants dans la mesure où une même firme peut approvisionner plusieurs concurrents.

\section{La capacité technologique de l'industrie}

Les dépenses de recherche industrielle de l'industrie aéronautique se sont chiffrées à $294 \mathrm{M} \$$ en 1982 , soit $12 \%$ des dépenses de R-D de l'industrie canadienne (Statistique Canada, cat. 88-202, tableau 5). Il faudrait ajouter les dépenses faites par le groupe des satellites. La recherche industrielle est fortement concentrée aux mains de quelques firmes. On peut estimer qu'environ $50 \%$ de la R-D de l'industrie aéronautique est faite par Pratt \& Whitney. En ce qui concerne plus spécifiquement l'industrie aérospatiale québécoise, $63 \%$ et $17 \%$ de tout le personnel de R-D se trouve respectivement chez Pratt \& Whitney et Spar Aerospace (Québec, CRIQ, 1983). Cinq entreprises québécoises ont au moins 5\% de leur personnel qui effectue des tâches de recherche industrielle: Pratt \& Whitney, Canadian Marconi, CAE Electronics, Aviation Electric et Spar Aerospace.

$\mathrm{Si}$ on situe la R-D comme un indicateur de l'activité de recherche, on doit d'abord conclure qu'il y a relativement peu de firmes dynamiques. Par exemple, alors que le Québec compte pour près de la moitié de la production de l'industrie, on ne 
retrouve que cinq entreprises qui effectuent un niveau minimal de recherche industrielle. Également, uniquement onze firmes canadiennes de l'aéronautique font de la R-D (Statistique Canada, cat. 88-202, tableau 25). S'il n'existait pas d'autres mécanismes pour obtenir la technologie, cet indicateur supposerait que la majorité des firmes de l'industrie ont une faible capacité technologique. Cependant, en ce qui concerne celles qui font de la $\mathrm{R}-\mathrm{D}$, elles en font à un niveau comparable à celui des firmes d'autres pays. En 1982, les entreprises de l'aéronautique qui faisaient de la R-D y ont alloué $14,5 \%$ de leurs ventes (Stastitique Canada, cat. 88-202, tableau 25). Il faut voir que la R-D n'est cependant qu'un indicateur de l'activité technologique d'une industrie. Cet indicateur suppose néanmoins que peu d'entreprises ont une production technologique autonome.

Les entreprises peuvent cependant développer une capacité technologique en procédant à des transferts plutôt qu'en produisant elles-mêmes la technologie. À cet égard, les firmes étrangères sont avantagées du fait qu'elles ont accès à la technologie développée à l'intérieur du réseau intra-firme. Pour les firmes sous contrôle canadien, cette stratégie est plus difficilement praticable. La soustraitance ne nécessite cependant pas de disposer de connaissances dans le domaine de l'aérospatiale. Le fabricant fournit à son soustraitant les spécifications des pièces faisant en sorte que son travail se résume souvent à fournir l'ingénierie. La sous-traitance reste alors une issue pour les entreprises ayant une faible capacité technologique. Toutefois, l'apprentissage qui résulte de ce type de production est très limité.

La capacité technologique de cette branche est donc relativement faible, bien qu'il y ait un noyau d'entreprises qui sont innovatrices. Ces entreprises occupent des créneaux sur le marché et leur production se fait en fonction d'une gamme de produits spécialisés. Ailleurs, il reste deux stratégies possibles: soit obtenir 
la technologie en bénéficiant des transferts, soit se limiter à la sous-traitance.

\section{Les politiques gouvernementales}

L'aérospatiale demeure l'une des priorités canadiennes en matière de développement technologique. Le tableau 5 présente les principales formes de dépense gouvernementale s'adressant aux entrerprises de ce secteur. L'aide de l'État passe principalement par quatre mécanismes.

\section{TABLEAU 5}

Principales mesures fédérales d'aide à l'industrie aérospatiale canadienne (million \$)

Contribution à la Corporation de développement des investissements du Canada - 1983-1984

Paiement à Canadair

Paiement à De Havilland

Subventions - Programme de productivité de l'industrie du matériel de défense - 1983-1984

- Entreprises recevant plus de $3 \mathrm{M \$}$

Pratt \& Whitney

$\$ 52,5$

Bell Helicopter

$\$ 12,3$

McDonnell Douglas

$\$ 6,6$

Spar Aerospace

$\$ 5,3$

Canadian Marconi

$\$ 4,9$

Canadair

$\$ 4,7$

Litton Systems

$\$ 3,6$

Achats gouvernementaux - Entreprises faisant partie de la liste des vingts premiers fournisseurs du Ministère des Approvisionnements et Services - 1982-1983

$\begin{array}{lr}\text { McDonnell Doublas, États-Unis } & \$ 126,8 \\ \text { Canadian Marconi } & \$ 123,0 \\ \text { Pratt \& Whitney } & \$ 118,5 \\ \text { Litton Systems } & \$ 91,1 \\ \text { Lockheed, États-Unis } & \$ 53,1 \\ \text { Spar Aerospace } & \$ 49,7 \\ \text { Bristol Aerospace } & \$ 46,4 \\ \text { Canadair } & \$ 45,0\end{array}$

Source: Comptes Publics, 1983-1984. Debra E. DOUGLAS, éd., How' to Sell to Governments in Canada, 1984, Don Mills, Corpus Information Services, 1984. 
Nationalisation. En étatisant Canadair et De Havilland au milieu des années soixante-dix, le gouvernement canadien a aussi voulu assurer la relance de l'industrie. Le programme Challenger chez Canadair visait à développer un appareil de technologie américaine avec des composants canadiens (Business and Commercial Aviation, 1983). En s'approvisionnant sur le marché canadien, Canadair devait favoriser la création d'une industrie en amont. Par la suite, occupant un créneau dans le domaine des jets d'affaires, l'entreprise devait écouler sa production sur le marché international et ainsi accroître les exportations canadiennes. L'aide gouvernementale passe alors par le financement des investissements et la «nationalisation» du risque.

Accords avec les États-Unis. La participation du gouvernement canadien au programme spatial américain représente une de ces formes d'accord. Le programme canado-américain de partage de la production de défense constitue un accord général en matière de production de matériel de défense: cet accord fait en sorte que les produits franchissent les frontières sans frais de douanes. On y prévoyait également que la technologie américaine devait être accessible aux firmes canadiennes. Sur ce dernier point, d'après les fabricants canadiens, l'entente ne donne par les résultats attendus (Canada, Rapport du Comité..., 1983: 7.4).

Achats gouvernementaux. Les gouvernements constituent le premier acheteur de matériel aérospatial dans tous les pays. Au Canada, huit entreprises de cette branche figurent sur la liste des vingt premiers fournisseurs du gouvernement fédéral. D’après le Ministère des approvisionnements et services, le gouvernement fédéral a acheté en 1983-84 30\% de la production aéronautique canadienne et $55 \%$ de la production québécoise de cette branche (Canada, A. et S. février 1985: 2). En 1979, les organismes gouvernementaux (incluant les gouvernements fédéral, provinciaux et locaux, de même que les sociétés d'État) ont acheté $14 \%$ de la production aéronautique canadienne. Ils ont plutôt consommé 
des produits importés: $72 \%$ de leurs achats étaient en matériel importé (Canada, A. et S., 1983). Dans le cas de Spar Aerospace, les achats gouvernementaux sont constitués en grande partie de commandes en recherche industrielle. En 1983-84, le gouvernement canadien a commandé des tâches en R-D à cette entreprise pour $25 \mathrm{M} \${ }^{11}$. Il s'agit de développer des produits qui seront par la suite fabriqués par cette entreprise. On peut estimer qu'environ $80 \%$ de la recherche industrielle effectuée par cette entreprise passe par de tels programmes ${ }^{12}$. Les achats gouvernementaux concernent également les retombées industrielles lorsque les achats sont faits hors du Canada ${ }^{13}$. Finalement, le gouvernement canadien investit dans l'industrie des satellites en contrats de R-D ou directement en achat de produits: en 1984-85, le Programme spatial devait représenter une dépense de $155 \mathrm{M} \$$ (Statistique Canada, cat. 88-204: 20).

Subventions. Le Programme de productivité de l'industrie du matériel de défense (P.P.I.M.D.) subventionne presque toutes les entreprises de cette branche. En $198231 \%$ de la R-D de l'industrie aéronautique de cette branche était financée par le gouvernement canadien. Alors que cette branche effectue $12 \%$ de toutes les dépenses de R-D de l'industrie canadienne, elle reçoit $31 \%$ du financement (Statistique Canada, 88-202, tableau 18). L'entreprise la plus subventionnée est Pratt \& Whitney. Les gouvernements se sont également engagés à verser $275 \mathrm{M} \$(165 \mathrm{M} \$$ et $110 \mathrm{M} \$$ par respectivement les gouvernements fédéral et québécois) pour assurer l'implantation de Bell Helicopter. Consécutivement à ce

11. Compilées d'après Canada, A. et $\mathrm{S}$., Bulletin ...

12. En 1983 et 1984 , Spar Aerospace déclare dépenser respectivement 4,8 M\$ et 5,5 M\$ en R-D.

13. McDonnel Douglas devait fournir des commandes aux entreprises canadiennes pour au moins 2,9 milliards $\$$, dont au moins $60 \%$ à l'industrie aéronautique et au moins $10 \%$ en transferts de technologie. En cas de non-respect de ces clauses, la pénalité était fixée à 120 M\$ (Canada, A. et S., 1981:10). 
projet, une subvention de $100 \mathrm{M} \$$ du gouvernement fédéral à Pratt \& Whitney est également prévue pour le développement et la fabrication des moteurs des hélicoptères.

Lorsqu'il s'agit de firmes sous contrôle étranger, les politiques gouvernementales visent à encourager l'attribution de mandats mondiaux (Canada, Conseil des Sciences, 1980 et Poynter et Rugman, 1982). À ce moment, la maison-mère octroie à sa filiale l'exclusivité du développement, de la production et de la vente de certains produits. Il faut signaler que les firmes actives sur les marchés publics et les plus subventionnées ont des mandats mondiaux.

La priorité gouvernementale qui apparaît dès le départ est le développement technologique et l'exportation. À cet égard, lorsqu'il s'agit de firmes étrangères, cette politique passe par l'octroi de mandats mondiaux aux filiales canadiennes. En effet, les mandats mondiaux sont devenus en quelque sorte une condition nécessaire pour obtenir l'aide gouvernementale, que ce soit en matière de subventions ou d'achats gouvernementaux.

Cependant, l'aide gouvernementale n'est pas entièrement orientée vers ces priorités. On aboutit alors à un paradoxe: alors que la stratégie est le soutien des entreprises de pointe, une bonne partie de la dépense gouvernementale dans cette branche va aussi à des entreprises moins dynamiques. L'État est en quelque sorte dans l'obligation de pallier l'instabilité du marché. Les gouvernements répondent alors à des pressions politiques.

\section{Spécialisation et contraintes}

L'industrie aérospatiale canadienne suit deux stratégies. L'une est la sous-traitance. Les firmes américaines sont ici privilégiées parce qu'elles ont un accès plus facile aux commandes des multinationales américaines. À l'extérieur du réseau intra-firme, il existe plusieurs concurrents sur les marchés étant donné que cette 
production est peu spécialisée. Les marchés sont aléatoires et il n'y a pas de transferts de technologie.

La seconde stratégie est de se spécialiser dans un créneau délaissé par les firmes de l'oligopole. Le choix de cette stratégie part du principe qu'il n'est pas possible de développer toute la gamme des produits aéronautiques en raison, d'une part, de la taille du marché national et, d'autre part, des ressources encore limitées de l'industrie canadienne. À un autre niveau, la stratégie de créneau s'appuie sur l'idée que la concurrence se fait au niveau de la technologie des produits (biens du «cycle du produit»). Dans ce contexte, l'innovation confere à l'entreprise un avantage comparatif lui permettant de distancier la concurrence et de monopoliser momentanément le marché. D’après cette logique, la stratégie de créneau passe par la sélection de produits sur lesquels il faut concentrer l'investissement. Il reste alors possible de faire l'innovation et de trouver un marché.

Cette stratégie prend des formes différentes suivant qu'elle est appliquée par une firme étrangère ou une firme canadienne. En ce qui concerne les firmes étrangères, il s'agit d'obtenir un mandat mondial: c'est-à-dire que l'entreprise décide que la filiale sera le seul fabricant d'une gamme de produits à l'intérieur du réseau intra-firme. Il est à noter que la filiale ne fait pas nécessairement le développement du produit, mais que la technologie peut provenir de la maison-mère qui laisse quand même à la filiale l'exclusivité de la fabricaiton. Une telle stratégie peut être décidée par la maison-mère qui choisit de rationaliser l'activité des filiales. La spécialisation permet notamment d'accroître les échelles de production. En ce qui concerne l'industrie aérospatiale, l'incitation provient davantage des politiques gouvernementales. L'enjeu est alors de faire en sorte que la filiale canadienne détienne l'exclusivité du produit. À ce moment, la filiale a accès au marché du réseau intra-firme ou au réseau de vente de la multinationale à travers le monde. Au départ, ces filiales ont la technologie de pointe et 
un marché permettant l'exportation qui est cependant limité par les règles de fonctionnement de la maison-mère. C'est donc au gouvernement que revient le rôle d'infléchir ces règles. Son impact dépend du poids de ces politiques sur la multinationale.

Pour les firmes sous contrôle canadien, il faut trouver des créneaux laissés vacants par les multinationales. Ces firmes n'ont cependant pas les deux avantages des firmes étrangères: la technologie et le marché du réseau intra-firme. Le cas de Challenger de Canadair est à cet égard un exemple typique. Ce projet s'appuyait sur des prévisions de marché et sur la faisabilité technologique de l'appareil. L'objectif était de développer un appareil s'adressant à une tranche spécifique du marché et ayant des propriétés différentes de la concurrence. Le développement de ce jet d'affaires implique d'abord des investissements en recherche industrielle, alors que l'entreprise n'est pas assurée de produire l'appareil intégrant les qualifications prévues au départ. Les coûts de développement du Challenger ont largement dépassé les premières estimations pour des performances moindres que celles attendues. De tels projets de recherche industrielle impliquent des risques en ce qui concerne la faisabilité technologique du projet. Il faut d'ailleurs souligner qu'une grande partie des pertes de Canadair sur ce projet provient de l'achat de pièces à des fournisseurs qui ne seront pourtant pas utilisées en raison de l'absence de marché ${ }^{14}$.

Il n'est pas possible de prévoir l'état de la concurrence et du marché. Il faut d'abord souligner que la fabrication des produits de haute technologie ne signifie pas automatiquement qu'il n'y a pas de concurrence. La concurrence s'avère très forte dans certains segments du marché. Par exemple, pour la fabrication des appareils court-courriers Dash-8, De Havilland fait maintenant face à cinq

14. En novembre 1982, devant le Comité permanent des finances, du Commerce et des questions économiques, les dirigeants de l'entreprise ont estimé entreposer dans leurs usines des pièces pour une valeur de $500 \mathrm{M} \$$. 
concurrents (tableau 3). Cette concurrence a débuté à partir du moment où tous les pays ont appliqué une politique de créneau similaire. On peut supposer que ces pays ont mis en place leur projet en raison, d'une part, de la capacité industrielle de leur pays et, d'autre part, de l'accès aux pièces demandant une plus forte capacité technologique. Ainsi, ces pays peuvent se permettre la production d'un appareil de cette taille, mais ne pourraient développer un plus grand appareil.

Cette stratégie de créneau traduit les tendances actuelles de la spécialisation internationale (Zarifian, 1983). C'est-à-dire que la concurrence dans les oligopoles internationaux passe de plus en plus par l'innovation. Ce phénomène est particulièrement visible dans l'industrie aérospatiale militaire où les prix sont secondaires par rapport aux propriétés des produits. Cette spécialisation implique aussi des coûts. Plusieurs firmes ont développé des avions qui n'ont ensuite jamais eu le marché prévu. On peut simplement rappeler les cas célèbres du Concorde et du Lockheed L-1011. Dans le dernier cas, l'entreprise américaine a surmonté la difficulté grâce aux commandes de l'armée.

La spécialisation fait en sorte que l'entreprise développe sa capacité industrielle pour une gamme très spécifique de produits et que sa production est ensuite intégrée à celle d'autres entreprises. Cette stratégie implique avant tout que cette gamme de produits soit toujours en demande. D'une part, dans ces secteurs de haute technologie, les bouleversements continuels découlant de l'innovation peuvent rendre ces produits désuets. D'autre part, ces créneaux existent dans la mesure où les oligopoles laissent à d'autres entreprises une fraction de la production et elles doivent donc continuer de le faire.

Cette spécialisation pose des contraintes sur le développement de l'industrie canadienne. Il y a d'abord une contrainte au niveau des approvisionnements. Il est rare que l'industrie parvienne à mettre sur le marché un produit fini complet et il faut alors y 
incorporer d'autres pièces généralement importées. Il faut donc que l'oligopole fabricant ces pièces accepte de les diffuser.

Cette contrainte est aussi présente au moment des transferts de technologie. Même pour les entreprises faisant l'innovation, il faut quand même avoir accès à la technologie américaine. Puisque la production de technologie au Canada est limitée et que la production va être intégrée à la production américaine, l'industrie doit s'appuyer sur la technologie américaine. Celle-ci est plus accessible pour les firmes étrangères. Étant donné les pratiques des firmes qui consistent à conserver le plus possible l'exclusivité de la technologie, elles permettent les transferts intra-firmes, mais beaucoup moins les transferts inter-firmes. En conséquence, dans la mesure où la production de l'industrie aérospatiale se fait à partir de la technologie américaine, les firmes américaines au Canada peuvent disposer d'un avantage sur leurs concurrents canadiens.

La vente sur le marché américain pose aussi quelques contraintes. Il faut signaler que ces contraintes ne proviennent pas du fait que la production est exportée, mais plutôt que l'exportation se fait sur un marché très imparfait en raison de la formation de l'oligopole et de la présence de l'État. Dans un marché aussi imparfait, c'est l'État qui a la charge de définir les marchés. Lorsque la production est exportée, l'Etat canadien n'a plus le pouvoir d'intervenir sur les marchés. L'industrie est donc dépendante des marchés étrangers et des décisions prises par les gouvernements. Il n'est donc plus possible de contrer certaines mesures néo-protectionnistes appliquées sur les marchés (Dalpé. 1984).

Les firmes étrangères peuvent plus facilement contourner ces contraintes. Elles vendent d'abord sur le marché plus stable du réseau intra-firme. C'est ainsi que les firmes qui approvisionnent le marché de l'aérospatiale civile aux Etats-Unis ont une plus grande stabilité que celles qui vendent des aéronefs civils sur ce 
même marché. Les firmes étrangères ont également certains avantages qui proviennent de la taille du réseau intra-firme. Par exemple, elles peuvent plus facilement répondre aux exigences des pays acheteurs que ne peuvent le faire les fabricants canadiens. Par exemple, face à certains pays exigeant des retombées industrielles lors des achats d'appareils, Canadair n'est pas concurrentiel dans certains pays.

\section{Conclusion}

Les contraintes auxquelles fait face l'industrie aérospatiale canadienne entraînent actuellement une plus forte intégration à l'industrie américaine. On assiste alors à un débordement de l'industrie américaine au nord de la frontière. En raison des nouvelles orientations moins interventionnistes que prend l'État canadien il faut s'attendre à ce que les entreprises autochtones laissent tomber les projets de grande ampleur. On peut prévoir que ces firmes vont s'orienter vers la sous-traitance de haut de gamme et vont coopérer à la fabrication d'appareils développés dans d'autres pays. Ces entreprises pourraient alors s'allier à un fabricant américain et s'occuper de la production d'une partie d'un appareil.

Le gouvernement canadien s'oriente maintenant vers une stratégie de mandats mondiaux. Ce rapprochement avec l'industrie américaine est viable tant que l'oligopole américain va conserver son leadership sur l'industrie occidentale. Cette politique se concrétise notamment par le soutien à Pratt \& Whitney (soutien direct par les subventions ou indirect par le biais des politiques d'implantation des deux usines d'hélicoptères, Bell Helicopter et le joint-venture canado-allemand Fleet Industries-MBBI ${ }^{15}$. Cette

15. Les gouvernements canadien et provincial ont versé des subventions pour l'implantation de ces deux usines d'hélicoptères. De plus, le gouvernement canadien verse directement à Pratt \& Whitney une subvention pour le développement des moteurs équipant ces hélicoptères. 
promotion des mandats mondiaux peut être efficace si le gouvernement canadien est prêt à soutenir ces entreprises par divers types de politiques. Dans l'industrie aérospatiale, l'attribution des mandats mondiaux dépend en grande partie de l'ampleur de l'aide gouvernementale que reçoivent les entreprises.

\section{Bibliographie}

BANQUE PARIBAS, «Les échanges technologiques mondiaux» Conjoncture, X11, no. 9, octobre 1982

Business and Commercial Aviation, septembre 1983, p. 37-41.

BOISMENU, Gérard, Robert DALPÉ et Graciela DUCATENZEILER, Quelques observations sur les transferts de technologie dans l'industrie manufacturière québécoise: étude de quatre branches industrielles, Communication à l'atelier de recherche sur l'économie et la politique de la technologie au Québec et au Canada, 19 mars 1985, Université de Montréal.

CANADA, Approvisionnements et Services, Bulletin des Marchés Publics, X11, no. 5, 14 février 1985.

CANADA, Approvisionnements et Services, Bulletin des Marchés Publics, X11, no. 7, 9 mai 1985

CANADA, Approvisionnements et Services, Bulletin Recherche et Développement.

CANADA, Approvisionnements et Services, Rapport Annuel 1980-81, 1981.

CANADA, Approvisionnements et Services, La Taille et la structure du marché du secteur public - 1979, 1983.

CANADA, Conseil des Sciences, Groupe d'étude de la politique industrielle, Les $\mathrm{Mul}$ tinationales et la stratégie industrielle, le rôle des droits exclusifs de diffusion mondiale d'un produit, Ottawa, Approvisionnements et Services, 1980.

CANADA, Conseil des Sciences, Les Transports et notre avenir énergétique, Rapport no 34, Ottawa, Approvisionnements et Services, 1982.

CANADA, Ministère de l'expansion économique régionale, Étude sur l'industrie aérospatiale canadienne dans le contexte nord-américain, 1981.

CANADA, Rapport du comité consultatif du développement de l'industrie aérospatiale. L'Aérospatiale du Canada - Point de vue et stratégie. Ottawa, Approvisionnements et Services, juin 1983.

DALPÉ, Robert, L'accès des firmes canadiennes du transport public et des télécommucinations aux marchés publics américains. Études internationales, XV, no 1, mars 1984 , p. $157-173$.

DOUGLAS WHITEHEAD CONSULTANTS, La Recherche sur les transports au Québec, Longueuil, l'Association québécoise du transport et des routes, mars 1983.

MORVAN, Yves, "L'Aéronautique", dans Bertrand BELLON et Jean-Marie CHEVALIER, éd., L'industrie en France, Paris, Flammarion, 1983, p. 251-279. 
MOWERY, David C, et Nathan ROSENBERG, "The commercial aircraft industry", dans Richard R. Nelson, éd., Government and Technical Progress - A Cross Industry Analysis, New York, Pergamon Press, 1982, p. 101-161.

MYTELKA, Lynn, "Le capitalisme fondé sur la connaissance et le changement dans les stratégies des entreprises industrielles", Études internationales, X1V, no 3, septembre 1983 , p. $433-452$.

PONSSARD, Jean-Pierre et Gérard De POURVOURVILLE, Marchés publics et politique industrielle, Paris, Economica, 1982.

POYNTER, Thomas A. et Alan M. RUGMAN, "World product mandates: how will multinationals respond?" Business Quarterly, vol. 47, no. 3, octobre 1982, p. 5461.

QUEBEC, Centre de recherche industrielle du Québec, Inventaire des entreprises manufacturières qui font de la recherche et du développement au Québec, Québec, Editeur officiel du Québec, 1983.

QUEBEC, Ministère de l'Industrie, du Commerce et du Tourisme, Matériel de transport - prévisions de la demande, évaluation de l'offre québécoise et des activités de recherche et de développement envisagées pour ces activités. Québec, janvier 1982.

Québec, Ministère des transports, Les Voies de l'avenir: la recherche et le développement en transport, Québec, Editeur officiel du Québec, 1983.

STATISTIQUE CANADA, Activités scientifiques fédérales 1984-85, Cat, 88-204.

STATISTIQUE CANADA, Statistiques sur la recherche et le développement industriels 1982, Cat. 88-202.

U.S., Department of Commerce, U.S. Industrial Outlook 1985, 1985.

Usine Nouvelle, 12 janvier 1984.

ZARIFIAN, Philippe, Le Redéploiement industriel, Paris, Le Sycomore, 1983. 